\title{
Sexual Contact as Risk Factor for Campylobacter Infection, Denmark
}

\author{
Katrin Gaardbo Kuhn, Anne Kathrine Hvass, Annette Hartvig Christiansen, Steen Ethelberg, Susan Alice Cowan
}

Campylobacteriosis is a disease of worldwide importance, but aspects of its transmission dynamics, particularly risk factors, are still poorly understood. We used data from a matched case-control study of 4,269 men who have sex with men (MSM) and 26,215 controls, combined with national surveillance data on Campylobacter spp., Salmonella spp., and Shigella spp., to calculate matched odds ratios (mORs) for infection among MSM and controls. MSM had higher odds of Campylobacter (mOR 14, 95\% Cl 10-21) and Shigella (mOR 74, 95\% Cl 27-203) infections, but not Salmonella (mOR 0.2, 95\% Cl 0-13), and were less likely than controls to have acquired Campylobacter infection abroad $\left(x^{2}=21 ; p<0.001\right)$. Our results confirm that sexual contact is a risk factor for campylobacteriosis and also suggest explanations for unique features of Campylobacter epidemiology. These findings provide a baseline for updating infection risk guidelines to the general population.

工 oodborne diseases are a global cause of illness -1 and death, imposing an economic burden on not only the food industry but also the public health sector. Campylobacter is the most frequently reported gastrointestinal bacterial pathogen in high-income countries (1), responsible for an estimated 166 million diarrheal illnesses worldwide and 3.7 million disability-adjusted life years (2). The disease is usually self-limiting, with symptoms manifesting as acute watery or bloody diarrhea; treatment is only required for severe cases. Campylobacter infection is a causal factor for Guillain-Barré syndrome, a peripheral nerve disorder, which can potentially cause paralysis. Incidence of Campylobacter infection is higher in men and boys than in women and girls, and

Author affiliations: University of Oklahoma Health Sciences Center, Oklahoma City, Oklahoma, USA (K.G. Kuhn); Statens Serum Institut, Copenhagen, Denmark (K.G. Kuhn, A.K. Hvass, A.H. Christiansen, S. Ethelberg, S.A. Cowan); University of Copenhagen Department of Public Health, Copenhagen (S. Ethelberg)

DOI: https://doi.org/10.3201/eid2704.202337 several countries report high incidence in children $<5$ years of age and in young adults $(1,3)$. In most high-income countries, infection with Campylobacter is notifiable as part of national surveillance programs for infectious diseases.

Campylobacteriosis is a zoonotic disease; poultry, wild birds, pets, and farm animals are the main reservoirs. Transmission to humans occurs primarily through unsafe handling or consumption of raw or undercooked chicken, consumption of raw milk, or contact with domestic animals (4-6). However, a large proportion of cases cannot be easily explained by these factors and it has been suggested that other infection routes (e.g., the environment) are equally important in explaining transmission of this disease $(4,7)$. Some zoonotic pathogens such as Shigella spp., Giardia lamblia, and Entamoeba histolytica have been associated with high risk for infection among men who have sex with men (MSM) because of anal-oral contact (8-12). Even though several outbreaks have been reported and observational studies have described a high incidence of Campylobacter infection among MSM $(8,13,14,15-$ $21)$, sexual contact is not officially considered among its risk factors for MSM or heterosexual partners in general. However, the transmission potential and incidence of gastrointestinal illnesses among MSM and heterosexual partners engaging in anal-oral sexual contact is difficult to evaluate based on laboratory data only, which does not contain sexual exposure information.

In Denmark, some infectious diseases, including all foodborne and most sexually transmitted infections, must be reported to Statens Serum Institut (SSI; https://en.ssi.dk), the national institute for infectious diseases of Denmark, through the national surveillance and notification system. The surveillance system comprises 2 parts: clinical notifications and laboratory notifications. Clinical notifications cover diseases that must be reported (Appendix, https:/ / wwwnc.cdc.gov/EID/article/27/4/20-2337-App1. pdf), including serious infectious diseases (e.g., 
meningitis and tuberculosis), sexually transmitted diseases, and Shigella spp. infections. These notifications include relevant patient information, primarily the unique individual Civil Registration System (CPR) number (22) and circumstances possibly affecting transmission of the infection, such as sexual contact, foreign travel, and contact with hospitals. General practitioners and hospital physicians fill out details on paper forms that are sent to SSI for manual entry into the database of the national clinical reporting system for infectious diseases. While MSM sexual contact is listed as a possible factor related to transmission, notifications do not include information on anal-oral contact between heterosexual partners.

The laboratory notification system receives reports of all gastrointestinal infections from certain microorganisms, including campylobacteriosis, salmonellosis, and shigellosis, for which clinical microbiological laboratories are obliged to report findings; reports also include the patient's CPR number. Laboratory notifications can include information on travel but this is not mandatory. Notifications of gastrointestinal infections are registered and stored in the Denmark Register of Enteric Pathogens. By using each patient's unique CPR number, duplication of patient records can be avoided and multiple reports for individual patients from the clinical infectious disease and enteric infections databases can be coupled. These data can then be used to generate linked datasets of notifiable diseases and possible explanatory or risk factors. We used these highquality national surveillance data in an individually matched case-control study undertaken to investigate the frequency of Campylobacter infections among MSM in Denmark.

\section{Methods}

\section{Study Design and Participants}

We undertook a national retrospective, individually matched case-control study, with a 9-year study period, 2010-2018, among men $\geq 18$ years of age residing in Denmark. Using an inverted case-control design, we considered MSM as case-patients and infections with Campylobacter spp., Shigella spp., or Salmonella spp. as exposures (Figure 1). We defined an MSM case-patient as a man $\geq 18$ years of age, with $\geq 1$ notification of any infectious disease (Appendix) acquired through MSM contact reported to SSI in a clinical notification during the study period. However, for men identified by CPR number in $>1$ notification during the study period, we included data from only 1 report. We excluded those $<18$ years of age at the time of the notification or with an incomplete CPR number. For the control group, we randomly selected men $\geq 18$ years of age from CPR digital records. We individually matched each MSM case-patient to (ideally) 3-5 controls by age (by year and month of birth) and municipality of residence. To determine exposures, we drew information on laboratory-confirmed infections with Campylobacter spp., nontyphoidal Salmonella spp., and Shigella spp. from the enteric infections database.

\section{Extraction of Data}

From the clinical infectious disease database, we extracted clinical notifications of men with an infectious disease acquired through MSM contact during 2010-2018, and from the enteric infections database, laboratory notifications of Campylobacter spp., Salmonella spp., and Shigella spp. infections over the same

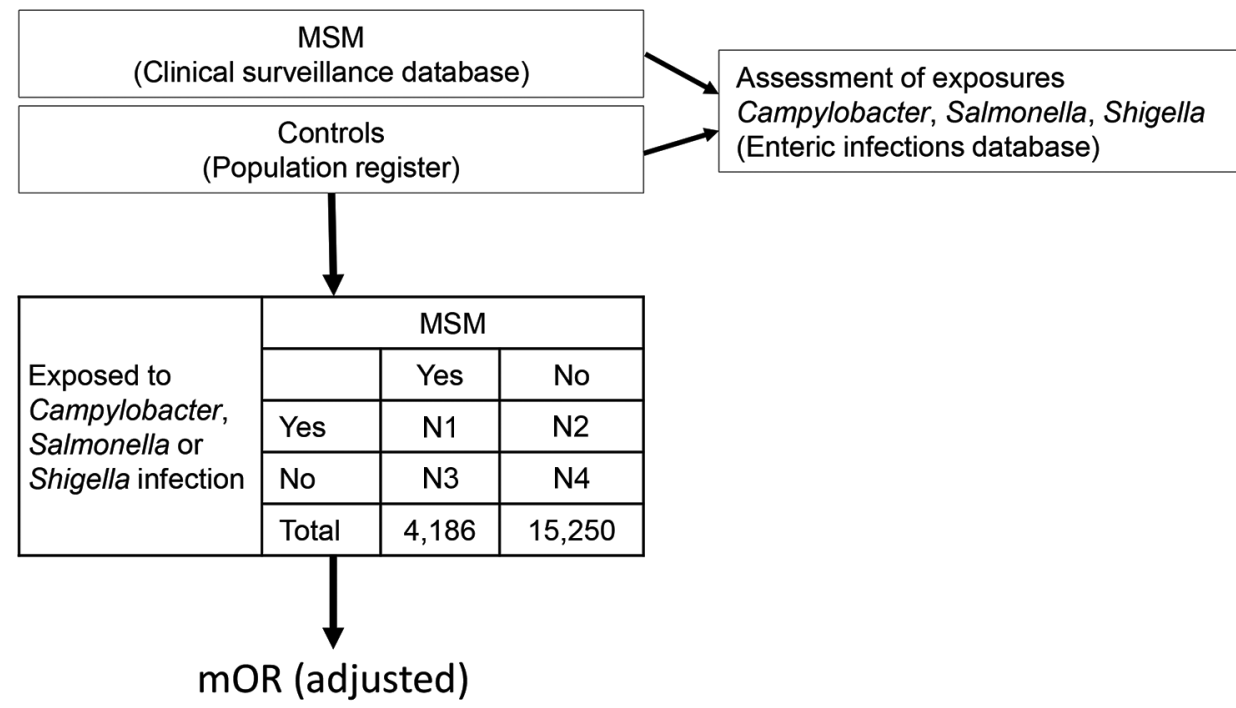

Figure 1. Inverted casecontrol study design in study of sexual contact as risk factor for Campylobacter infection, Denmark, 2010-2018. mOR adjusted for foreign travel, year of notification, infection with any of the other pathogens, and recurrent infections. mOR, matched odds ratio; MSM, men who have sex with men. 
period. For each disease notification, we obtained the CPR number of the MSM case-patient, year of notification, and whether the infection was assumed to have been acquired in Denmark or abroad. We included notifications for all species of the 3 pathogens. Data on campylobacteriosis, salmonellosis, and shigellosis were extracted for both sexes and all age groups but only reports for men $\geq 18$ years of age at the time of notification were included in the study. For calculation of age-specific incidence of the pathogens, we used national population data available from Statistics Denmark (https:/ / www.statistikbanken.dk).

\section{Calculations and Statistical Analysis}

To illustrate the age distribution of infection, we divided case-patients and controls into 2 groups: those $<40$ years of age and those $\geq 40$ years of age. We analyzed the difference between the frequency of travelacquired and recurrent infections in the MSM and control groups, age distributions of infections, and the geographic distribution of MSM case-patients in comparison to the general population using $\chi^{2}$ tests. We calculated unadjusted and adjusted matched odds ratios (mORs) with 95\% CIs for the 3 exposures, Campylobacter, Salmonella, and Shigella infections, in MSM case-patients and controls using conditional logistic regression. The analysis was adjusted for foreign travel, year of notification, infection with any of the other pathogens included in the study, and recurrent infections. We analyzed the distribution of MSM notifications and gastrointestinal diseases over time using a simple regression for trend. We performed all data analyses using Stata version 14 (StataCorp, https://www.stata.com).
This study was approved under the general agreement for noninterventional database studies between the Danish Data Protection Agency and Statens Serum Institut (reference number 2008-54-0474). According to regulations in Denmark, ethics committee approval is not required for studies that do not involve analysis of biologic material from human subjects.

\section{Results}

From the clinical infectious disease database, we extracted 4,269 individual reports (only 1 per person) of men who had acquired a notifiable disease through MSM contact during January 1, 2010-December 31, 2018. Of these, $83(1.9 \%)$ men were excluded because they did not have either a valid CPR number or an address in Denmark. For the control group, we extracted 15,250 randomly selected matched male controls from the CPR registry. The mean age was 41 years (median 40 years, range 18-88 years) for both the case-patients and the controls. Case-patients and controls were geographically distributed throughout Denmark, but a significantly larger proportion of the study population than the general population resided in the Capital Region $\left(\chi^{2}=1,400 ; p<0.0001\right)$.

From the Register of Enteric Pathogens, we extracted 49,321 notifications of infections with $\geq 1$ of the 3 bacterial pathogens (exposures); 748 patients had registrations for $\geq 2$ recurrent infections with the same pathogen during the study period (Table 1). Most $(76 \%)$ notifications were for Campylobacter infections: $55 \%$ C. jejuni, $4 \%$ C. coli, and $40 \%$ other Campylobacter spp. with no species reported. In the MSM group, a total of 132 Campylobacter, 3 Salmonella, and

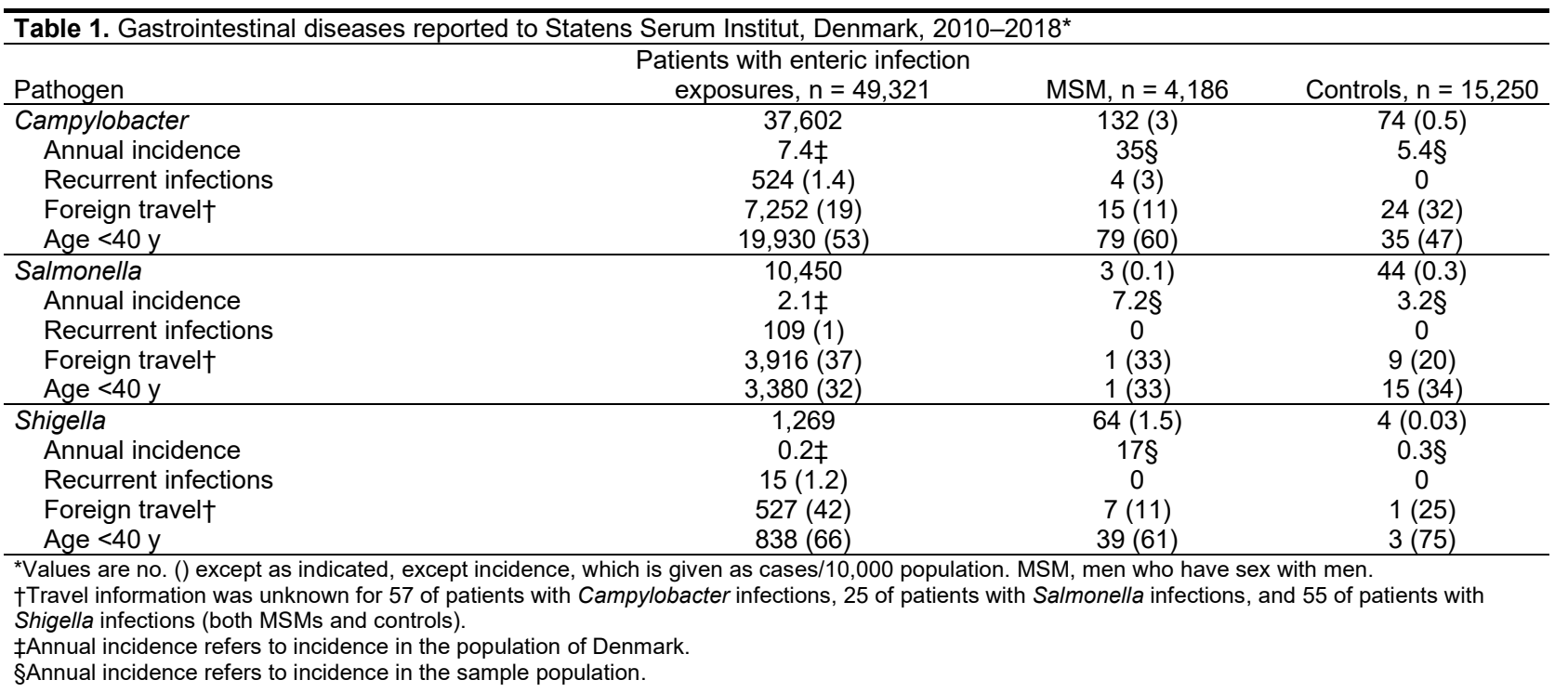


64 Shigella infections were reported during the study period (Table 1). In the control group, we observed 74 Campylobacter, 44 Salmonella, and 4 Shigella infections (Table 1). Compared with controls, a higher proportion of MSM case-patients $<40$ years of age had Campylobacter infections (Table 1), although this difference was not statistically significant $\left(\chi^{2}=3 ; p=0.08\right)$.

Overall, we found that the odds of a Campylobacter infection were 14 times higher among MSM than controls (Table 2). MSM case-patients also had 74 times higher odds than controls of being infected with Shigella, a pathogen known to be transmitted by sexual contact (Table 2). However, we found no significant difference between Salmonella infection rates in MSM case-patients and controls (Table 2). MSM case-patients who were infected with Campylobacter were significantly less likely to have acquired their infection abroad compared with controls $\left(\chi^{2}=21 ; \mathrm{p}<0.001\right)$, which was not the case for Salmonella or Shigella. Over the study period, there were $4(3 \%)$ recurrent Campylobacter infections in the MSM group compared with none in the control group (statistical analysis not possible); there were no recurrent Salmonella or Shigella infections in the MSM or control groups. During the study period, we observed an increase among MSM case-patients in clinical infections acquired through MSM contact and Campylobacter or Shigella infections (Figure 2, $t=5-11 ; p<0.001)$. We did not observe any change in the proportion of Salmonella among MSM case-patients $(t=-2 ; p=0.1)$ or for any of the 3 pathogens in the control group (Figure 2, $\mathrm{t}=-1$ to $2 ; \mathrm{p}=0.1-0.4$ ).

\section{Discussion}

We present surveillance data-driven evidence that campylobacteriosis can be transmitted through sexual contact. Among our study group of MSM casepatients, the odds of being infected with Campylobacter was 14 times higher than among controls. We observed a similar pattern for shigellosis, which is known to be transmitted by sexual contact, but not for Salmonella infection. In Denmark, Salmonella infections are considered almost exclusively foodborne whereas domestic Shigella infections are regarded as primarily sexually transmitted and secondarily as foodborne or transmitted through general person-to-person contact. Campylobacter infection is linked to several different transmission routes (4), handling or consuming of poultry long considered the most notable.

However, our results reinforce information from many reports suggesting Campylobacter can be transmitted through sexual contact; these reports provide explanations for unique aspects of Campylobacter epidemiology supported by biologic facts from other foodborne bacteria. Reported outbreaks among MSM in Canada $(14,19,23,24)$ and previous observations of higher infection rates in homosexual men and HIV-positive patients $(8,13,17,20,21,25,26)$ indicate the likelihood that Campylobacter can be transmitted through sexual contact. In spite of this, sexual contact has not traditionally been considered a possible transmission route in sporadic campylobacteriosis cases and therefore has been excluded as a possible risk factor in published case-control studies (4). We were unable to assess the risk of Campylobacter transmission through anal-oral contact between heterosexual partners because only MSM sexual contact is reported on notifications. However, it is highly likely that the risk of infection through this type of sexual contact is equally relevant for heterosexual and MSM partners.

Several aspects of Campylobacter epidemiology remain to be clarified, such as why the disease is more common among men (3,13,27-29). Many explanations have been proposed, including differences in food handling and preparation, healthcare-seeking behaviors, and physiologies. We provide an additional explanation: adult men practicing sex with other men are at significantly higher risk $(\mathrm{p}<0.001)$ of campylobacteriosis. In this study, Campylobacter incidence in the MSM group was almost 5 times higher than in the general population, which could be a partial driver for higher incidence in men.

Another feature observed in surveillance statistics from several countries was the biphasic age distribution of Campylobacter infections, showing pronounced peaks in children $<5$ years of age and young adults $20-40$ years of age $(3,30-33)$. In the 20-40-year age group, these peaks have also been observed for Shigella $(34,35)$, but not for other foodborne bacteria

Table 2. Matched odds ratios by gastrointestinal infection among MSM and controls in study of sexual contact as risk factor for Campylobacter infection, Denmark, 2010-2018*

\begin{tabular}{|c|c|c|c|c|c|c|}
\hline \multirow[b]{2}{*}{ Pathogen } & \multirow{2}{*}{$\begin{array}{c}\text { MSM, no. (\%), } \\
n=4,186\end{array}$} & \multirow{2}{*}{$\begin{array}{c}\text { Controls, no. (\%), } \\
n=15,250\end{array}$} & \multicolumn{2}{|c|}{ Unadjusted comparison } & \multicolumn{2}{|c|}{ Adjusted comparison† } \\
\hline & & & $\mathrm{mOR}(95 \% \mathrm{Cl})$ & $p$ value & $\mathrm{mOR}(95 \% \mathrm{Cl})$ & $p$ value \\
\hline Campylobacter & $132(3)$ & $74(0.5)$ & $16(11-23)$ & $<0.001$ & $14(10-21)$ & $<0.001$ \\
\hline Salmonella & $3(0.07)$ & $5(0.03)$ & $3(0.7-13)$ & 0.132 & $0.2(0.02-1.3)$ & 0.09 \\
\hline Shigella & $64(1.5)$ & $4(0.03)$ & $105(37-307)$ & $<0.001$ & $74(27-203)$ & $<0.001$ \\
\hline
\end{tabular}

${ }^{*}$ mOR, matched odds ratio; MSM, men who have sex with men.

†Adjusted for foreign travel, year of notification/infection, infection with any of the other pathogens and recurrent infections. 
Figure 2. Percentages of clinical notifications of infections acquired through MSM contact (notifiable infections) and Campylobacter, Salmonella, and Shigella infections reported among MSM and controls in study of sexual contact as risk factor for Campylobacter infection, Denmark, 2010-2018. MSM were men $\geq 18$ years of age notified of any infectious disease acquired through sexual contact with another man. Controls were men $\geq 18$ years randomly selected from the Denmark population register. MSM and controls < 18 years of age or who did not have a valid national civil registration number were excluded from the study. MSM, men who have sex with men.

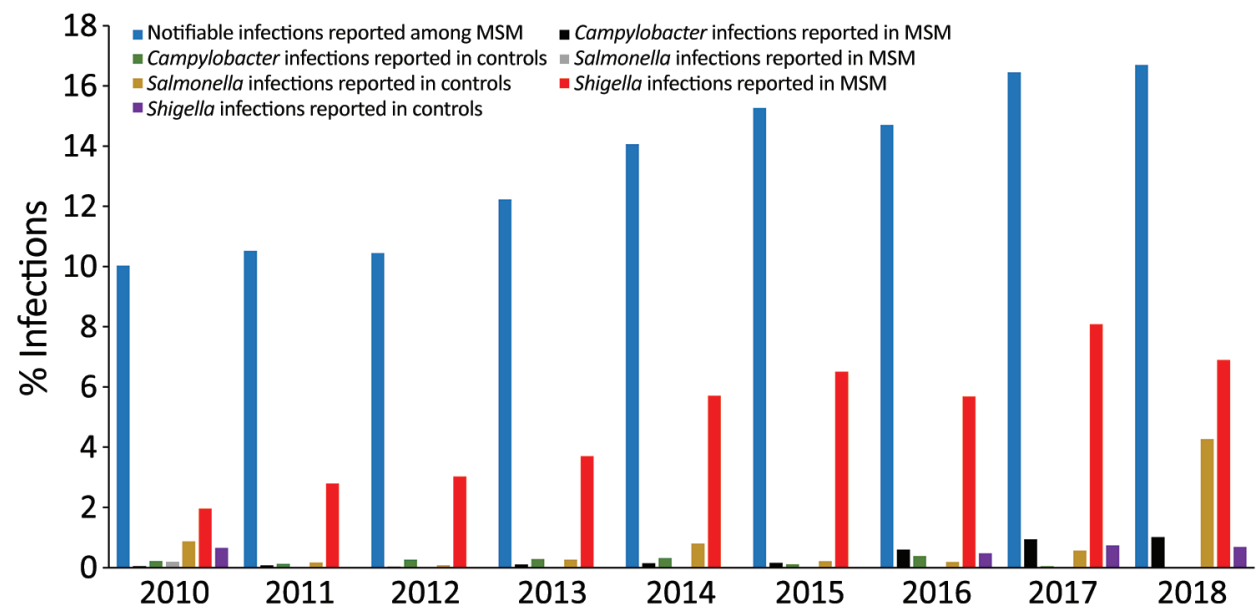

such as Salmonella. These findings have been explained by the secondary weaning phase, meaning that young adults away from home are less aware of proper hygiene practices, secondary infections from caring for young children at home, or hormonal and behavioral factors. When comparing different age groups, we saw higher Campylobacter infection rates in MSM $<40$ years of age compared with controls, although this difference was not statistically significant because of the small sample size. On the basis of our results, we suggest that, in countries with a clear biphasic Campylobacter age distribution, some of the high infection frequency among young adults might be explained by sexual transmission among persons in this very sexually active age group.

Bacterial pathogens can be transmitted through fecal-oral contact during sex, either directly, through anal-oral contact or anal-penile-oral contact, or indirectly, such as through the use of sex toys or fingers. In 1974, sexual contact was recognized as a risk factor for infection with Shigella spp. (11), and such contact is now widely acknowledged as one of the most important transmission routes for shigellosis. The probability of infection with a foodborne bacterium from fecal-oral contact is directly related to the infectious dose. For Shigella spp. this dose can be as low as 10 1,000 organisms, and for Campylobacter, 500-10,000 organisms (36). The infectious dose for Salmonella spp. varies (37) but might be as high as 1 million bacterial cells. Pathogens with a low infectious dose are easier to transmit from person to person, including through sexual contact; on the basis of our findings, we tentatively propose that this might be the case for Shigella and Campylobacter, but not Salmonella.
In Denmark and the rest of Europe, campylobacteriosis and shigellosis are often acquired abroad; $30 \%-50 \%$ of infections are reported as travel related $(3,33)$. This possibility was reflected in our control group, but Campylobacter infections among MSM were significantly less likely to have been travel related $(p<0.001)$. Assuming that many Campylobacter and Shigella cases in the MSM group have been acquired by sexual contact, this domestic pattern is not surprising because MSM contact is most commonly not a holiday experience and infection is more likely to happen at home.

During the study period, there was a significant $(p<0.001)$ increase in the proportion of MSM case-patients infected with Shigella or Campylobacter, but not with Salmonella; we found no increase in the control group. Incidence of campylobacteriosis in Denmark has increased recently (3), reflecting a combination of actual increases in cases of the disease, changes in diagnostic techniques, and improved electronic notification. During the study period, laboratory diagnosis of gastrointestinal pathogens has transitioned from culturing to more sensitive PCR testing of feces. This change could explain some of the increase in recorded Shigella and Campylobacter infections; however, similar increases would be expected for Salmonella and for the number of infections in the control group. An increasing trend over time would also be observed if there had been larger outbreaks of Shigella and Campylobacter among MSM case-patients later in the study period, but only 1 outbreak of shigellosis was reported, and none of campylobacteriosis among MSM during this time (38). The most likely explanation for the observed increase in Shigella and Campy- 
lobacter among MSM case-patients is the concurrent increase in the proportion of infections transmitted through MSM contact reported to SSI. More extensive reporting has increased the amount of data available in the electronic databases, in turn increasing the likelihood of discovering infections in the MSM group.

Our study had 2 major strengths: use of routinely collected high-quality national surveillance data for our analyses and results supported by biologic evidence and demographic information, such as the infectious doses and the low level of travel-related infections in the MSM group that explain some of the unique characteristics of Campylobacter transmission. The first limitation is that, although clinical notifications of diseases in Denmark can include MSM contact as possibly associated with the circumstances of the infection, such inclusions are based on patient statements, and only HIV, syphilis, gonorrhea, and hepatitis infections must be reported. Therefore, the MSM population group probably did not include all men with an infection acquired through MSM contact during the study period. It is also very likely that the control group included some MSM and that some reported Campylobacter, Shigella, or Salmonella infections in the control group were acquired through MSM contact. However, such an underestimation would tend to lower the estimates of association toward 1 and, in fact, approximate the odds ratios to risk ratios. Differences between case-patients and controls concerning how exposure was determined (i.e., from a notification to SSI about infection with an enteric pathogen) could have affected the results. However, because physicians and laboratories in Denmark operate under the same guidelines and procedures and the notification records are uniformly registered, such differences are unlikely to have had an effect.

The results in this study provide a measure of the risk associated with a notifiable infectious disease among a specific group of MSM and not among a general MSM population. However, considering the robust mORs and corresponding $\mathrm{CIs}$ combined with published reports about high Campylobacter incidence in MSM populations, we believe that our findings can be extrapolated to MSM in general. The case-patient group might overrepresent risk-taking persons more likely to practice unsafe sex, and if that is the case, the increased risk for Campylobacter infection through sexual contact would primarily apply to this highrisk group. However, it can also be argued that this risk applies to the wider MSM population because condom use would not eliminate the infection risk associated with several fecal-oral transmission routes. The case-patients we selected might also have a high frequency of known risk factors, such as use of proton pump inhibitors, certain occupations, or increased contact with animals, that we could not account for in this study.

Another limitation of the data used in our study was the incomplete travel information for persons with reported infections. Travel history was unknown for patients with 25\% of Salmonella, 55\% of Shigella, and $57 \%$ of Campylobacter infections because of incomplete registration and lack of follow-up interviews. This proportion was similar between casepatients and controls. In spite of this, our results still indicated that MSM are less likely to have acquired Campylobacter or Shigella infections abroad. Also, although our data covered all infections reported as acquired through MSM contact and all confirmed cases of campylobacteriosis, salmonellosis, and shigellosis, these do not account for all cases. Notifications are based on passive surveillance and underestimate the true incidence because persons with infections who do not seek medical attention are not captured (39). However, because our system enabled linkage of individual data collected, we believe that these results are as accurate as possible and could be generalized to similar settings. Lack of association between MSM contact and Salmonella infection might reflect the true situation or that a larger sample size is needed.

The cycle of transmission through sexual contact among adults contributes substantially to the burden of disease in highly industrialized countries, but likely not in low-middle income settings where limited hygiene and sanitation constitute greater risk factors. Therefore, the external validity of the results in some locations should be considered and we encourage public health agencies in other countries to replicate this study to corroborate the findings. Finally, as we did not directly measure Campylobacter infection transmitted through anal-oral contact, the association is speculative and our conclusions are derived from surveillance data analyses in combination with knowledge gained from studies of other bacteria.

Our findings indicate a strong likelihood that Campylobacter can be transmitted during sexual contact. Given previous reports of outbreaks and high incidence of Campylobacter among MSM, this is not surprising. Combining this theory with high-quality national surveillance data, our results offer additional reasonable explanations for why surveillance statistics from some countries show that adult men are more frequently infected with Campylobacter than are women and why Campylobacter incidence peaks among young adults. Overall, our findings not only 
address epidemiologic questions but also highlight a need to inform the public about the risk of infection through sexual activity in regions where Campylobacter incidence exhibits patterns similar to those in Denmark. These results are primarily applicable to adults in high-income settings in some countries; further studies are warranted among the general MSM population rather than only those with a reported notifiable disease. However, considering the high burden of campylobacteriosis in Europe, the United States, and Australia, incorporating knowledge from these findings into general information campaigns might encourage the use of precautionary measures during sexual contact, which could, in turn, lead to lower infection rates and reduced overall costs to society.

\section{Acknowledgments}

We are grateful to Kåre Mølbak and Tyra Grove Krause for feedback on the study.

\section{About the author}

Dr. Kuhn is a senior infectious disease epidemiologist working as a professor at the University of Oklahoma Health Sciences Center. Her research interests include foodborne and waterborne infections, climate change, and research related to One Health collaborations.

\section{References}

1. Kaakoush NO, Castaño-Rodríguez N, Mitchell HM, Man SM. Global epidemiology of Campylobacter infection. Clin Microbiol Rev. 2015;28:687-720. https://doi.org/ 10.1128/CMR.00006-15

2. Havelaar AH, Kirk MD, Torgerson PR, Gibb HJ, Hald T, Lake RJ, et al.; World Health Organization Foodborne Disease Burden Epidemiology Reference Group. World Health Organization global estimates and regional comparisons of the burden of foodborne Disease in 2010. PLoS Med. 2015;12:e1001923. https:/ / doi.org/10.1371/ journal.pmed.1001923

3. Kuhn KG, Nielsen EM, Mølbak K, Ethelberg S. Epidemiology of campylobacteriosis in Denmark 2000-2015. Zoonoses Public Health. 2018;65:59-66. https://doi.org/10.1111/ zph.12367

4. Kuhn KG, Nielsen EM, Mølbak K, Ethelberg S. Determinants of sporadic Campylobacter infections in Denmark: a nationwide case-control study among children and young adults. Clin Epidemiol. 2018;10:1695-707. https:/ / doi.org/ 10.2147/CLEP.S177141

5. MacDonald E, White R, Mexia R, Bruun T, Kapperud G, Lange $\mathrm{H}$, et al. Risk factors for sporadic domestically acquired Campylobacter infections in Norway 2010-2011: a national prospective case-control study. PLoS One. 2015;10:e0139636. https:/ / doi.org/10.1371/journal. pone.0139636

6. Mughini Gras L, Smid JH, Wagenaar JA, de Boer AG, Havelaar AH, Friesema IHM, et al. Risk factors for campylobacteriosis of chicken, ruminant, and environmental origin: a combined case-control and source attribution analysis. PLoS One. 2012;7:e42599. https:/ / doi.org/10.1371/ journal.pone.0042599

7. Hansson I, Sandberg M, Habib I, Lowman R, Engvall EO. Knowledge gaps in control of Campylobacter for prevention of campylobacteriosis. Transbound Emerg Dis. 2018;65(Suppl 1):30-48. https:// doi.org/10.1111/tbed.12870

8. Narayan S, Galanis E; BC STEI Group. Are enteric infections sexually transmitted in British Columbia? Can Commun Dis Rep. 2016;42:24-9. https:// doi.org/10.14745/ ccdr.v42i02a01

9. Escobedo AA, Almirall P, Alfonso M, Cimerman S, Chacín-Bonilla L. Sexual transmission of giardiasis: a neglected route of spread? Acta Trop. 2014;132:106-11. https://doi.org/10.1016/j.actatropica.2013.12.025

10. Fletcher SM, Stark D, Harkness J, Ellis J. Enteric protozoa in the developed world: a public health perspective. Clin Microbiol Rev. 2012;25:420-49. https:/ / doi.org/10.1128/ CMR.05038-11

11. Tauxe RV, McDonald RC, Hargrett-Bean N, Blake PA. The persistence of Shigella flexneri in the United States: increasing role of adult males. Am J Public Health. 1988;78:1432-5. https:// doi.org/10.2105/ AJPH.78.11.1432

12. Mildvan D, Gelb AM, William D. Venereal transmission of enteric pathogens in male homosexuals. Two case reports. JAMA. 1977;238:1387-9. https://doi.org/10.1001/ jama.1977.03280140065022

13. Mook P, Gardiner D, Kanagarajah S, Kerac M, Hughes G, Field N, et al. Use of gender distribution in routine surveillance data to detect potential transmission of gastrointestinal infections among men who have sex with men in England. Epidemiol Infect. 2018;146:1468-77. https://doi.org/10.1017/S0950268818001681

14. Marchand-Senécal X, Bekal S, Pilon PA, Sylvestre J-L, Gaudreau C. Campylobacter fetus cluster among men who have sex with men, Montreal, Quebec, Canada, 2014-2016. Clin Infect Dis. 2017;65:1751-3. https:/ / doi.org/10.1093/ $\mathrm{cid} / \mathrm{cix} 610$

15. Gaudreau C, Rodrigues-Coutlée S, Pilon PA, Coutlée F, Bekal S. Long-lasting outbreak of erythromycin- and ciprofloxacin-resistant Campylobacter jejuni subspecies jejuni from 2003 to 2013 in men who have sex with men, Quebec, Canada. Clin Infect Dis. 2015;61:1549-52. https://doi.org/10.1093/cid/civ570

16. Allos BM, Allos BM. Campylobacter jejuni infections: update on emerging issues and trends. Clin Infect Dis. 2001;32: 1201-6. https:/ / doi.org/10.1086/319760

17. Sorvillo FJ, Lieb LE, Waterman SH. Incidence of campylobacteriosis among patients with AIDS in Los Angeles County. J Acquir Immune Defic Syndr (1988). 1991;4:598-602.

18. Quinn TC, Goodell SE, Fennell C, Wang SP, Schuffler MD, Holmes KK, et al. Infections with Campylobacter jejuni and Campylobacter-like organisms in homosexual men. Ann Intern Med. 1984;101:187-92. https:// doi.org/10.7326/0003-4819101-2-187

19. Gaudreau C, Michaud S. Cluster of erythromycin- and ciprofloxacin-resistant Campylobacter jejuni subsp. jejuni from 1999 to 2001 in men who have sex with men, Québec, Canada. Clin Infect Dis. 2003;37:131-6. https://doi.org/10.1086/375221

20. Newman KL, Newman GS, Cybulski RJ, Fang FC. Gastroenteritis in men who have sex with men in Seattle, Washington, 2017-2018. Clin Infect Dis. 2020;71:17. https:/ /doi.org/10.1093/cid/ciz783 
21. Gerninger AL, Addetia A, Starr K, Cybulski RJ, Stewart MK, Salipante SJ, et al. International spread of multidrugresistant Campylobacter coli in men who have sex with men in Washington state and Quebec, 2015-2018. Clin Infect Dis. 2020;71:29. https://doi.org/10.1093/cid/ciz1060

22. Pedersen CB. The Danish Civil Registration System. Scand J Public Health. 2011;39(7 Suppl):22-5. https://doi.org/10.1177/1403494810387965

23. Gaudreau C, Pilon PA, Sylvestre J-L, Boucher F, Bekal S. Multidrug-resistant Campylobacter coli in men who have sex with men, Quebec, Canada, 2015. Emerg Infect Dis. 2016;22:1661-3. https:// doi.org/10.3201/eid2209.151695

24. Gaudreau C, Helferty M, Sylvestre J-L, Allard R, Pilon PA, Poisson M, et al. Campylobacter coli outbreak in men who have sex with men, Quebec, Canada, 2010-2011. Emerg Infect Dis. 2013;19:764-7. https://doi.org/10.3201/eid1905.121344

25. Hughes G, Silalang P, Were J, Patel H, Childs T, Alexander S, et al. Prevalence and characteristics of gastrointestinal infections in men who have sex with men diagnosed with rectal chlamydia infection in the UK: an 'unlinked anonymous' cross-sectional study. Sex Transm Infect. 2018;94:518-21. https:/ / doi.org/10.1136/ sextrans-2016-053057

26. Larsen IK, Gradel KO, Helms M, Hornstrup MK, Jürgens G, Mens $\mathrm{H}$, et al. Non-typhoidal Salmonella and Campylobacter infections among HIV-positive patients in Denmark. Scand J Infect Dis. 2011;43:3-7. https:/ / doi.org/10.3109/00365548.20 10.517780

27. Walter F, Ott JJ, Claus H, Krause G. Sex- and age patterns in incidence of infectious diseases in Germany: analyses of surveillance records over a 13-year period (2001-2013). Epidemiol Infect. 2018;146:372-8. https://doi.org/10.1017/ S0950268817002771

28. Moffatt CRM, Glass K, Stafford R, D'Este C, Kirk MD. The campylobacteriosis conundrum - examining the incidence of infection with Campylobacter sp. in Australia, 1998-2013. Epidemiol Infect. 2017;145:839-47. https://doi.org/10.1017/ S0950268816002909

29. Strachan NJC, Watson RO, Novik V, Hofreuter D, Ogden ID, Galán JE. Sexual dimorphism in campylobacteriosis. Epidemiol Infect. 2008;136:1492-5. https:/ / doi.org/10.1017/ S0950268807009934

30. Gillespie IA, O’Brien SJ, Penman C, Tompkins D, Cowden J, Humphrey TJ. Demographic determinants for Campylobacter infection in England and Wales: implications for future epidemiological studies. Epidemiol Infect. 2008;136:1717-25. https://doi.org/10.1017/S0950268808000319
31. Schielke A, Rosner BM, Stark K. Epidemiology of campylobacteriosis in Germany - insights from 10 years of surveillance. BMC Infect Dis. 2014;14:30. https:/ / doi.org/ 10.1186/1471-2334-14-30

32. Geissler AL, Bustos Carrillo F, Swanson K, Patrick ME, Fullerton KE, Bennett C, et al. Increasing Campylobacter infections, outbreaks, and antimicrobial resistance in the United States, 2004-2012. Clin Infect Dis. 2017;65:1624-31. https:// doi.org/10.1093/cid/cix624

33. Nichols GL, Richardson JF, Sheppard SK, Lane C, Sarran C. Campylobacter epidemiology: a descriptive study reviewing 1 million cases in England and Wales between 1989 and 2011. BMJ Open. 2012;2:e001179. https://doi.org/10.1136/ bmjopen-2012-001179

34. Haley CC, Ong KL, Hedberg K, Cieslak PR, Scallan E, Marcus R, et al. Risk factors for sporadic shigellosis, FoodNet 2005. Foodborne Pathog Dis. 2010;7:741-7. https:/ / doi.org/10.1089/fpd.2009.0448

35. European Centre for Disease Prevention and Control. Shigellosis - annual epidemiological report 2016 (2014 data). Stockholm: The Centre; 2016 [cited 2019 May 3]. http:/ / ecdc.europa.eu/en/publications-data/shigellosisannual-epidemiological-report-2016-2014-data

36. Kothary MH, Babu US. Infective dose of foodborne pathogens in volunteers: a review. J Food Saf. 2001;21:49-68. https://doi.org/10.1111/j.1745-4565.2001.tb00307.x

37. Blaser MJ, Newman LS. A review of human salmonellosis: I. infective dose. Rev Infect Dis. 1982;4:1096-106. https://doi.org/10.1093/clinids/4.6.1096

38. Statens Serum Institut. Epi-News: week 48 - 2017. Shigellose 2014-2016. Copenhagen: Statens Serum Institut [cited 2021 Jan 1]. https:/ / www.ssi.dk/Aktuelt/Nyhedsbreve/ EPI-NYT/2017/Uge\%2048\%20-\%202017

39. Haagsma JA, Geenen PL, Ethelberg S, Fetsch A, Hansdotter F, Jansen A, et al.; Med-Vet-Net Working Group. Community incidence of pathogen-specific gastroenteritis: reconstructing the surveillance pyramid for seven pathogens in seven European Union member states. Epidemiol Infect. 2013;141:1625-39. https:/ / doi.org/ $10.1017 /$ S0950268812002166

Address for correspondence: Katrin Gaardbo Kuhn, Hudson College of Public Health, University of Oklahoma Health Sciences Center, 801 NE 13th St, Oklahoma City, OK 73104, USA; email: katrin-kuhn@ouhsc.edu 